\title{
On-Line Estimation of All Electrical Parameters in Induction Machines subject to Stator Fault
}

\author{
Mikael Stocks ${ }^{\dagger}$ and Alexander Medvedev $\ddagger$
}

\begin{abstract}
Identification of all the electrical parameters of Induction Machines (IMs) is a prerequisite for successful modelbased control and/or fault detection. Most of the existing identification methods of symmetrical IMs are, strictly speaking, valid only at stationary conditions, which limitation results in either poor dynamics excitation or gross approximation errors, depending on the working conditions of the motor.

In this paper, the topic of identification of all electrical parameters of faulty IMs is addressed for the first time. A method for on-line observer-based estimation of all electrical parameters of IMs with faulty stator is presented aiming primarily at fault tolerant control and fault detection. The approach does not rely on any assumptions regarding the rotor angular speed and/or acceleration.
\end{abstract}

\section{NOMENCLATURE}

Variables

$\psi \quad$ - magnetic flux

- electrical current

- feeding voltage

- rotor speed
Parameters

$R_{A}^{\prime}, R_{S} \quad$ - electrical resistances

$L_{A}^{\prime}, L_{S}^{\prime} \quad$ - lumped inductances

$M_{A r}^{\prime}, M \quad$ - mutual inductances

$\varepsilon_{A}, \varepsilon_{S}, \varepsilon_{r} \quad$ - electrical parameters

$\mu_{A}, \mu \quad$ - goodness factors

$K_{A}, K_{S} \quad$ - normalization factors

For the variables, - denotes a normalized (dimensionless) variable and subscripts $\alpha$ and $\beta$ refers to the corresponding axis in a two-axis system, respectively.

For both the variables and parameters, capital letter subscript $A$ refers to stator axis $A$ in a conventional three-axis system. Similarly, subscript $S$ refers to stator axis $B$ or $C$. In the same manner, a small letter subscript $r$ refers to rotor axis $a, b$ or $c$. The units used for the variables and parameters are SI-units throughout the paper.

Bold capital letters stand for matrices, whereas small and capital letters denote respectively scalars and vectors. Identity and zero matrices of appropriate dimension (mostly $2 \times 2$ ) are denoted as $\mathbf{I}$ and $\mathbf{0}$. The unitary matrix

$$
\mathbf{J}=\left[\begin{array}{cc}
0 & -1 \\
1 & 0
\end{array}\right]
$$

is frequently used in the sequel. The complex conjugate transpose of a matrix is denoted by ${ }^{H}$ and ${ }^{*}$ means complex conjugate of a scalar. The determinant of a matrix is denoted by $|\cdot|$. The transition matrix of matrix $\mathbf{A}$ is denoted by $\boldsymbol{\Phi}_{\mathbf{A}}\left(t, t_{0}\right)$.

${ }^{\dagger}$ Systems and Interaction, Luleå University of Technology, SE-971 87 Luleå, SWEDEN, e-mail: Stocks@ltu.se

¥Information Technology, Uppsala University, SE-751 05 Uppsala, SWEDEN, e-mail: Alexander.Medvedev@it.uu.se

\section{INTRODUCTION}

Induction machines (IMs) are widely used today in various applications. For example, it is by far the most used electrical motor in industry today. Thus, there is a need for efficient methods of on-line parameter identification of IMs to facilitate motor fault detection and condition monitoring. The subject is though mathematically challenging, since the IM dynamics are time-varying, due to the varying speed of the rotor.

Several on-line methods for estimation of all parameters of the healthy IM have been proposed, mostly based on various Linear Regression models estimated by Least Squares techniques. In all such approaches, approximations have been utilized to make the motor dynamics fit the identification framework. In continuous time identification, it is convenient to neglect the rotor acceleration, $i$. $e$. assume the rotor angular velocity to be constant. However, such a simplification compromises the accuracy of parameter identification. At those time instants when the approximation holds, the IM usually operates at or close to stationary conditions where excitation is poor, which results in incorrect parameter identification results. On the other hand, when the excitation is rich, $i$. $e$. at rapidly changing rotational speed of the rotor, the approximation is not justified, and the errors for the estimated parameters may become large. Moreover, the first and second order derivatives of the stator voltages and currents are also often needed, which calls for filtering of these quantities. Filtering causes several negative effects as well. If not performed carefully, severe phase-shifts are introduced, especially during important transients with rich excitation.

The drawbacks described above lead altogether to questionable identification results, see for instance [1] and [2]. An attempt to minimize the impact of poor excitation is reported in [3], where Total Least Squares is employed for parameter estimation. The results indicate that the problems experienced with Ordinary Least Squares may be alleviated, although not cured.

Linear regression models are also applied in discrete time, [4], [5]. Similarly to continuous time, this approach has problems with poor excitation under stationary conditions and inadequate modeling under transients. In addition, all measured variables are typically assumed to be constant between sampling instants, which results in poor approximation since both the feeding voltages and the stator currents for an uncontrolled motor are typically sinusoidal and during transients the rotational speed of the rotor varies. Curing this shortcoming calls for shorter sampling period, which 
leads to higher computational burden and more pronounced measurement noise.

An observer-based approach to parameter identification is presented in [6], where the original state vector has been extended with some auxiliary states and a full order linear observer is designed. It works in continuous time and does not use any common devastating assumptions as these in the continuous time LS techniques. However, for the symmetrical IM, the underlying dynamical model used is of higher order compared to the algorithm presented in this paper.

No parameter identification techniques for faulty IMs have been found by the authors in the literature. It is most likely the case because of the lack of relevant parsimonious mathematical models of asymmetrical (faulty) IMs. However, there is a sharp need for parameter identification for such machines, mainly because it opens up for the possibility of fault detection with tight bounds, and even more important, allows to distinguish between different kinds of faults. In [7], it is shown that different stator faults manifest themselves in different patterns of parameter alternations in a newly developed model of faulty IMs, hence discrimination between different kind of faults becomes possible.

In this paper, a continuous observer-based method for online estimation of all electrical parameters in IMs subject to stator fault is presented. First, a low-order dynamic model of the IM with a stator fault is summarized. Then, the problem of model parameter estimation is solved by means of a recently developed optimal observer with guaranteed convergence rate. Numerical simulations confirm efficacy of the suggested method.

\section{Model OF Induction Machines}

A normalized model of the electrical part of an IM with faulty stator (see [7],[8]) can be written as

$$
\begin{aligned}
\mu_{A} \dot{\bar{\tau}}_{\alpha S} & =\dot{\bar{\psi}}_{\alpha S}-\varepsilon_{r}\left(\bar{\imath}_{\alpha S}-\bar{\psi}_{\alpha S}\right)-\bar{\omega} \frac{M_{A r}^{\prime}}{M}\left(\mu \bar{\imath}_{\beta S}-\bar{\psi}_{\beta S}\right) \\
\mu \dot{\bar{\tau}}_{\beta S} & =\dot{\bar{\psi}}_{\beta S}-\varepsilon_{r}\left(\bar{\imath}_{\beta S}-\bar{\psi}_{\beta S}\right)+\bar{\omega} \frac{M}{M_{A r}^{\prime}}\left(\mu_{A} \bar{\imath}_{\alpha S}-\bar{\psi}_{\alpha S}\right) \\
\dot{\bar{\psi}}_{\alpha S} & =-\varepsilon_{A} \bar{\imath}_{\alpha S}+\bar{u}_{\alpha S} ; \dot{\bar{\psi}}_{\beta S}=-\varepsilon_{S} \bar{\imath}_{\beta S}+\bar{u}_{\beta S}
\end{aligned}
$$

The output map to the measured stator currents is given by

$$
\imath_{\alpha S}=K_{A} \bar{\imath}_{\alpha S} ; \imath_{\beta S}=K_{S} \bar{\imath}_{\beta S}
$$

It is assumed that all parameters in (1) and (2) are constant and unknown. The rotor speed $\bar{\omega}$ is in sequel treated as a perfectly known time-varying parameter. Note that eight independent coefficients are needed in order to describe the dynamics of the IM; $K_{A}, K_{S}, \mu_{A}, \mu, \varepsilon_{A}, \varepsilon_{S}, \varepsilon_{r}$ and the quotient $\frac{M_{A r}^{\prime}}{M}$, which seems to be the least number.

In what follows,

$$
\imath_{S}=\left[\begin{array}{c}
\imath_{\alpha S} \\
\imath_{\beta S}
\end{array}\right] ; x_{S}=\left[\begin{array}{c}
x_{\alpha S} \\
x_{\beta S}
\end{array}\right] ; z_{S}=\left[\begin{array}{c}
z_{\alpha S} \\
z_{\beta S}
\end{array}\right]
$$

Now, assume that $\imath_{\alpha S}, \imath_{\beta S}, \bar{\omega}, \bar{u}_{\alpha S}$ and $\bar{u}_{\beta S}$ are perfectly known, $i$.e measured without noise. Taking into account the expressions for $x_{\alpha S}, x_{\beta S}$

$$
\begin{aligned}
x_{\alpha S} & =\frac{K_{S}}{K_{A}} \frac{M}{M_{A r}^{\prime}} \frac{\mu_{A}}{\mu} \frac{K_{A}}{\mu_{A}} \bar{\psi}_{\alpha S}=\frac{M}{M_{A r}^{\prime}} \frac{K_{S}}{\mu} \bar{\psi}_{\alpha S} \\
x_{\beta S} & =\frac{K_{A}}{K_{S}} \frac{M_{A r}^{\prime}}{M} \frac{\mu}{\mu_{A}} \frac{K_{S}}{\mu} \bar{\psi}_{\beta S}=\frac{M_{A r}^{\prime}}{M} \frac{K_{A}}{\mu_{A}} \bar{\psi}_{\beta S}
\end{aligned}
$$

and introducing

$$
z_{S}=\varepsilon_{r}\left[\begin{array}{cc}
\frac{K_{A}}{K_{S}} \frac{M_{A r}^{\prime}}{M} \frac{\mu}{\mu_{A}} & 0 \\
0 & \frac{K_{S}}{K_{A}} \frac{M}{M_{A r}^{\prime}} \frac{\mu_{A}}{\mu}
\end{array}\right] x_{S}
$$

model (1), with the use of (2) to transform the currents, is transformed into a time-varying linear form

$$
\begin{aligned}
i_{S} & =-\bar{\omega} \mathbf{J} x_{S}+z_{S}+\bar{\omega} \mathbf{J} \tilde{\mathbf{A}}_{\mathbf{1 1}} \theta_{1}+\tilde{\mathbf{A}}_{\mathbf{1 2}} \theta_{2} \\
\dot{x}_{S} & =\tilde{\mathbf{A}}_{\mathbf{1 2}} \theta_{3} \\
\dot{z}_{S} & =\tilde{\mathbf{A}}_{\mathbf{1 2}} \theta_{4} \\
\dot{\theta}_{1} & =\dot{\theta}_{2}=\dot{\theta}_{3}=\dot{\theta}_{4}=0
\end{aligned}
$$

where

$$
\begin{aligned}
& \tilde{\mathbf{A}}_{11}=\left[\begin{array}{cc}
\imath_{\alpha S} & 0 \\
0 & \imath_{\beta S}
\end{array}\right] \\
& \tilde{\mathbf{A}}_{12}=\left[\begin{array}{cccc}
\bar{u}_{\alpha S} & 0 & -\imath_{\alpha S} & 0 \\
0 & \bar{u}_{\beta S} & 0 & -\imath_{\beta S}
\end{array}\right] \\
& \theta_{1}=\left[\begin{array}{ll}
\frac{K_{S}}{K_{A}} \frac{M}{M_{A r}^{\prime}} \frac{\mu_{A}}{\mu} & \frac{K_{A}}{K_{S}} \frac{M_{A r}^{\prime}}{M} \frac{\mu}{\mu_{A}}
\end{array}\right]^{T} \\
& \theta_{2}=\left[\begin{array}{llll}
\frac{K_{A}}{\mu_{A}} & \frac{K_{S}}{\mu} & \frac{\varepsilon_{A}}{\mu_{A}}+\frac{\mu}{\mu_{A}} \frac{\varepsilon_{r}}{\mu} & \frac{\varepsilon_{S}}{\mu}+\frac{\varepsilon_{r}}{\mu}
\end{array}\right]^{T} \\
& \theta_{3}=\left[\begin{array}{llll}
\frac{M}{M_{A r}^{\prime}} \frac{K_{S}}{\mu} & \frac{M_{A r}^{\prime}}{M} \frac{K_{A}}{\mu_{A}} & \theta_{33} & \theta_{34}
\end{array}\right]^{T} \\
& \theta_{4}=\varepsilon_{r}\left[\begin{array}{llll}
\frac{K_{A}}{\mu_{A}} & \frac{K_{S}}{\mu} & \frac{\varepsilon_{A}}{\mu_{A}} & \frac{\varepsilon_{S}}{\mu}
\end{array}\right]^{T}
\end{aligned}
$$

and

$$
\begin{aligned}
\theta_{33} & =\frac{K_{S}}{K_{A}} \frac{M}{M_{A r}^{\prime}} \frac{\mu_{A}}{\mu} \frac{\varepsilon_{A}}{\mu_{A}} \\
\theta_{34} & =\frac{K_{A}}{K_{S}} \frac{M_{A r}^{\prime}}{M} \frac{\mu}{\mu_{A}} \frac{\varepsilon_{S}}{\mu}
\end{aligned}
$$

The extended system has two more dynamic states and 14 more static states compared to (1), amounting to a total increase of 16 . Hence, the system has been augmented by eight more states than the number of the original independent coefficients. This redundancy is the price to pay for enabling linear observer-based parameter estimation without approximations.

\section{OBSERVER DESIGN}

In standard state-space notation, model (3) has the linear time-varying form of

$$
\begin{aligned}
& \dot{x}=\mathbf{A} x \\
& y=\mathbf{C} x
\end{aligned}
$$

For the observer

$$
\begin{aligned}
\dot{\hat{x}} & =\mathbf{A} \hat{x}+\mathbf{K}(y-\hat{y}) \\
\hat{y} & =\mathbf{C} \hat{x}
\end{aligned}
$$


the error system has the following structure,

$$
\begin{aligned}
{\left[\begin{array}{c}
\dot{\tilde{z}} \\
\tilde{\theta}
\end{array}\right] } & =\left[\begin{array}{cc}
\mathbf{A}_{11}-\mathbf{K}_{\mathbf{1}} \mathbf{C}_{\mathbf{1}} & \mathbf{A}_{\mathbf{1 2}} \\
-\mathbf{K}_{\mathbf{2}} \mathbf{C}_{\mathbf{1}} & \mathbf{0}
\end{array}\right]\left[\begin{array}{c}
\tilde{z} \\
\tilde{\theta}
\end{array}\right] \\
& =\left[\begin{array}{cc}
\mathbf{A}_{11} & \mathbf{A}_{\mathbf{1 2}} \\
\mathbf{0} & \mathbf{0}
\end{array}\right]\left[\begin{array}{c}
\tilde{z} \\
\tilde{\theta}
\end{array}\right]-\left[\begin{array}{c}
\mathbf{K}_{1} \\
\mathbf{K}_{\mathbf{2}}
\end{array}\right] r \\
r & =\left[\begin{array}{ll}
\mathbf{C}_{\mathbf{1}} & \mathbf{0}
\end{array}\right]\left[\begin{array}{c}
\tilde{z} \\
\tilde{\theta}
\end{array}\right] ; e=\left[\begin{array}{c}
\tilde{z} \\
\tilde{\theta}
\end{array}\right]
\end{aligned}
$$

where $\tilde{z}$ corresponds to errors in the dynamical states, and $\tilde{\theta}$ to errors in static states, i.e. parameters. In (7), the matrices are

$$
\begin{aligned}
\mathbf{A}_{11} & =\left[\begin{array}{ccc}
0 & -\bar{\omega} \mathbf{J} & \mathbf{I} \\
0 & 0 & 0 \\
0 & 0 & 0
\end{array}\right] \\
\mathbf{A}_{12} & =\left[\begin{array}{cccc}
\bar{\omega} \mathbf{J} \tilde{\mathbf{A}}_{11} & \tilde{\mathbf{A}}_{12} & 0 & 0 \\
0 & 0 & \tilde{\mathbf{A}}_{12} & 0 \\
0 & 0 & 0 & \tilde{\mathbf{A}}_{12}
\end{array}\right] \\
\mathbf{C}_{1} & =\left[\begin{array}{lll}
\mathbf{I} & 0 & 0
\end{array}\right]
\end{aligned}
$$

where all relevant matrix blocks can be identified from (3).

To design stable observers corresponding to (7), one might first try the simplest possible approach, the Algebraic Lyapunov Equation (ALE). Unfortunalely, as the theorem below proves, such a simple approach is not possible, due to the process dynamics.

Theorem 1: Assume $\imath_{S}, \bar{u}_{S} \in \mathcal{R}^{2}$ and $\bar{\omega} \in \mathcal{R}$. Consider a Lyapunov function candidate for (7) in the form of $V=$ $e^{H} \mathbf{P} e$, where $\mathbf{P} \in \mathcal{C}^{n \times n}$ is a hermitian, positive definite constant matrix, i. e. $\mathbf{P}>\mathbf{0}, \dot{\mathbf{P}}=\mathbf{0}$. Then,

$$
\mathbf{P A}+\mathbf{A}^{H} \mathbf{P}=-\mathbf{Q}
$$

does not hold for any admissible $\mathbf{P}, \mathbf{Q} \geq \mathbf{0}$.

Proof: See [9].

In the light of this result, a more powerful and general for linear time varying systems technique is sought. Following [10], consider the feedback matrix $\mathbf{K}$ given by

$$
\begin{aligned}
\mathbf{K} & =\mathbf{P C}^{H} \mathbf{Q} \\
\dot{\mathbf{P}} & =\alpha \mathbf{P}+\mathbf{A P}+\mathbf{P A}^{H}-\mathbf{P} \mathbf{C}^{H} \mathbf{Q} \mathbf{C P}+\mathbf{G G}^{H}
\end{aligned}
$$

where $\mathbf{Q}(t)$ is chosen so that

$$
\mathbf{C}^{H} \mathbf{Q C} \triangleq \mathbf{H}^{H} \mathbf{H} \geq \mathbf{0}
$$

and the time-varying matrix $\mathbf{G}$ is of suitable dimensions. Error system (7) can then be written as

$$
\begin{aligned}
\dot{e} & =\left(\mathbf{A}-\mathbf{P} \mathbf{C}^{H} \mathbf{Q} \mathbf{C}\right) e \\
& =\mathbf{A} e-\mathbf{P} \mathbf{C}^{H} \mathbf{Q} r \\
\dot{\mathbf{P}} & =\alpha \mathbf{P}+\mathbf{A P}+\mathbf{P} \mathbf{A}^{H}-\mathbf{P} \mathbf{C}^{H} \mathbf{Q} \mathbf{C P}+\mathbf{G G}^{H} \\
r & =\mathbf{C} e
\end{aligned}
$$

where $e\left(t_{0}\right)=e_{0}$ and $\mathbf{P}\left(t_{0}\right)=\mathbf{P}_{\mathbf{0}}$. The following two theorems summarize relevant properties of the resulting observer. Definitions of (uniform) observability/controllability on an interval are needed for their formulation. Consider

$$
\begin{aligned}
\dot{x} & =\mathbf{A} x+\mathbf{B} u \\
y & =\mathbf{C} x+\mathbf{D} u
\end{aligned}
$$

where $x \in \mathcal{C}^{n}, u \in \mathcal{C}^{m}$ and $y \in \mathcal{C}^{\ell}$. In the sequel, the timevarying complex-valued matrices $\mathbf{A}, \mathbf{B}, \mathbf{C}$ and $\mathbf{D}$, as well as the input and output $u, y$, are assumed perfectly known during each time-interval of interest. The state-transition matrix $\mathbf{\Phi}_{\mathbf{A}}\left(t, t_{0}\right)$ of (11) is the unique solution to

$$
\dot{\mathbf{\Phi}}_{\mathbf{A}}\left(t, t_{0}\right)=\mathbf{A}(t) \boldsymbol{\Phi}_{\mathbf{A}}\left(t, t_{0}\right) ; \quad \mathbf{\Phi}_{\mathbf{A}}\left(t_{0}^{-}, t_{0}^{-}\right)=\mathbf{I}
$$

Definition 1: System (11) is said to be observable on $\mathcal{I}=$ $\left[t_{0}, t_{f}\right]$ if the observability gramian

$$
\mathcal{O}\left(t_{0}, t_{f}\right)=\int_{t_{0}^{-}}^{t_{f}^{+}} \boldsymbol{\Phi}_{\mathbf{A}}^{H}\left(\tau, t_{0}\right) \mathbf{C}^{H}(\tau) \mathbf{C}(\tau) \boldsymbol{\Phi}_{\mathbf{A}}\left(\tau, t_{0}\right) d \tau
$$

is positive definite. Analogously, (11) is said to be controllable on $\mathcal{I}$ if the controllability gramian

$$
\mathcal{C}\left(t_{0}, t_{f}\right)=\int_{t_{0}^{-}}^{t_{f}^{+}} \boldsymbol{\Phi}_{\mathbf{A}}\left(t_{0}, \tau\right) \mathbf{B}(\tau) \mathbf{B}^{H}(\tau) \boldsymbol{\Phi}_{\mathbf{A}}^{H}\left(t_{0}, \tau\right) d \tau
$$

is positive definite.

Dealing with time-varying systems, Definition 1 is seldom sufficient for observer (or controller) design. Therefore, more restrictive notions are often used.

Definition 2: System (11) is said to be uniformly observable on $\mathcal{I}$ if for some $\delta_{o} \geq 0$ the two following conditions hold

$$
\begin{gathered}
\alpha_{l} \mathbf{I} \leq \mathcal{O}\left(T, T+\delta_{o}\right) \leq \alpha_{u} \mathbf{I}, \quad t_{0} \leq T \leq t_{f}-\delta_{o} \\
\left\|\mathbf{\Phi}_{\mathbf{A}}(t, \tau)\right\|_{p} \leq f(|t-\tau|),
\end{gathered}
$$

for some $\alpha_{l}, \alpha_{u}>0$ and $f(\cdot): \mathcal{R} \rightarrow \mathcal{R}$ is bounded on bounded intervals. The pair $(\mathbf{C}, \mathbf{A})$ is then said to be a uniformly observable pair. Similarly, (11) is said to be uniformly controllable on $\mathcal{I}$ if for some $\delta_{c} \geq 0$ the two following conditions hold

$$
\begin{array}{cc}
\beta_{l} \mathbf{I} \leq \mathcal{C}\left(T, T+\delta_{c}\right) \leq \beta_{u} \mathbf{I}, & t_{0} \leq T \leq t_{f}-\delta_{c} \\
\left\|\mathbf{\Phi}_{\mathbf{A}}(t, \tau)\right\|_{p} \leq f(|t-\tau|), & t, \tau \in \mathcal{I}
\end{array}
$$

for some $\beta_{l}, \beta_{u}>0$. Similarly, $(\mathbf{A}, \mathbf{B})$ is then said to be a uniformly controllable pair.

Theorem 2 (Bounded positive definite solution):

Assume that for some constant $\alpha_{\max }$

$$
|\alpha(t)| \leq \alpha_{\max }, \quad \alpha(t) \in \mathcal{R}, t \in \mathcal{I}
$$

$(\mathbf{H}, \mathbf{A})$ is a uniformly observable pair on $\mathcal{I}$ and $\mathbf{P}_{\mathbf{0}}>\mathbf{0}$. In addition, let at least one of the following alternatives hold:

I: $\quad(\mathbf{A}, \mathbf{G})$ is a uniformly controllable pair on $\mathcal{I}$

II: the system $\dot{\xi}=\left(\mathbf{A}+\frac{1}{2} \alpha \mathbf{I}\right) \xi$ is diverging so that for $K, \beta>0$

$$
\left\|\boldsymbol{\Phi}_{\left(\mathbf{A}+\frac{1}{2} \alpha \mathbf{I}\right)}^{-1}\left(t, t_{0}\right)\right\|_{p} \leq K e^{-\beta\left(t-t_{0}\right)}
$$

Then

$$
c_{l} \mathbf{I} \leq \mathbf{P}(t) \leq c_{u} \mathbf{I}, \quad t \in \mathcal{I}
$$

for some $c_{l}, c_{u}>0$.

Proof: See [10].

Theorem 3 (Exponential Convergence): Assume that the conditions of Theorem 2 hold and $\alpha(t) \geq \alpha_{\min } \geq 0, t \in$ 
$\mathcal{I}$ for some constant $\alpha_{\min }$. Then a solution to (10) converges exponentially at a rate of

$$
\|e(t)\| \leq \lambda^{\prime} e^{-\frac{1}{2} \gamma\left(t, t_{0}\right)} \leq \lambda e^{-\frac{1}{2} \alpha_{\min }\left(t-t_{0}\right)}, t \in \mathcal{I}
$$

for some $\lambda^{\prime}, \lambda \geq 0$ and

Proof: See [10].

$$
\gamma\left(t, t_{0}\right)=\int_{t_{0}}^{t} \alpha(\tau) d \tau
$$

The Theorems above provide the basis for observer-based parameter identification presented in the sequel.

\section{Estimating Parameters From States}

Estimated states of the extended systems can be used for $e$. g. parameter identification and/or fault detection. In this respect, the estimated static states are used to obtain conventional IM parameters. The following result is a suboptimal solution for the model describing faulty stator dynamics.

Theorem 4: Consider the observer (6) designed for (5). Define $\vartheta=\left[\begin{array}{cc}\theta_{2}^{T} & \theta_{4}^{T}\end{array}\right]^{T}$. Assume $\hat{\theta}_{i j}>0$. Define $\bar{\vartheta}=$ $\hat{\vartheta}-\vartheta\left(\hat{K}_{A}, \hat{K}_{S}, \hat{\mu}_{A}, \hat{\mu}, \hat{\varepsilon}_{A}, \hat{\varepsilon}_{S}, \hat{\varepsilon}_{r}\right)$ and $V=\bar{\vartheta}^{H} \bar{\vartheta}$. Then the unique solution to $\min V: \hat{\varepsilon}_{r}>0$ is given by

$$
\begin{aligned}
\hat{K}_{A} & =\frac{\hat{\theta}_{41} \hat{\varepsilon}_{r}+\hat{\theta}_{21}}{\hat{\theta}_{23} \hat{\varepsilon}_{r}-\hat{\theta}_{43}} \frac{\hat{\varepsilon}_{r}^{2}}{1+\hat{\varepsilon}_{r}^{2}} \\
\hat{K}_{S} & =\frac{\hat{\theta}_{42} \hat{\varepsilon}_{r}+\hat{\theta}_{22}}{\hat{\theta}_{24} \hat{\varepsilon}_{r}-\hat{\theta}_{44}} \frac{\hat{\varepsilon}_{r}^{2}}{1+\hat{\varepsilon}_{r}^{2}} \\
\hat{\mu}_{A} & =\frac{\hat{\varepsilon}_{r}^{2}}{\hat{\theta}_{23} \hat{\varepsilon}_{r}-\hat{\theta}_{43}} ; \hat{\mu}=\frac{\hat{\varepsilon}_{r}^{2}}{\hat{\theta}_{24} \hat{\varepsilon}_{r}-\hat{\theta}_{44}} \\
\hat{\varepsilon}_{A} & =\frac{\hat{\theta}_{43} \hat{\varepsilon}_{r}}{\hat{\theta}_{23} \hat{\varepsilon}_{r}-\hat{\theta}_{43}} ; \hat{\varepsilon}_{S}=\frac{\hat{\theta}_{44} \hat{\varepsilon}_{r}}{\hat{\theta}_{24} \hat{\varepsilon}_{r}-\hat{\theta}_{44}} \\
\hat{\varepsilon}_{r} & =a+\sqrt{a^{2}+1}
\end{aligned}
$$

where

$$
a=\frac{1}{2} \frac{\left(\hat{\theta}_{41}^{2}+\hat{\theta}_{42}^{2}\right)-\left(\hat{\theta}_{21}^{2}+\hat{\theta}_{22}^{2}\right)}{\hat{\theta}_{21} \hat{\theta}_{41}+\hat{\theta}_{22} \hat{\theta}_{42}}
$$

and $\frac{\partial \hat{\varepsilon}_{r}}{\partial \hat{\theta}_{23}}=\frac{\partial \hat{\varepsilon}_{r}}{\partial \hat{\theta}_{24}}=\frac{\partial \hat{\varepsilon}_{r}}{\partial \hat{\theta}_{43}}=\frac{\partial \hat{\varepsilon}_{r}}{\partial \hat{\theta}_{44}}=0$. Furthermore, if $\tilde{\vartheta}=$ $\vartheta-\hat{\vartheta}$ is sufficiently "small", then $\hat{K}_{A}, \hat{K}_{S}, \hat{\mu}_{A}, \hat{\mu}, \hat{\varepsilon}_{A}, \hat{\varepsilon}_{S}>0$. Finally, $\tilde{\vartheta}=0 \Leftrightarrow \hat{K}_{A}=K_{A}, \hat{K}_{S}=K_{S}, \hat{\mu}_{A}=\mu_{A}, \hat{\mu}=$ $\mu, \hat{\varepsilon}_{A}=\varepsilon_{A}, \hat{\varepsilon}_{S}=\varepsilon_{S}, \hat{\varepsilon}_{r}=\varepsilon_{r}$.

Proof: See [9].

Theorem 4 can be used to sub-optimally estimate all but one of the model parameters of an IM with faulty stator. Assume that $\hat{K}_{A}, \hat{K}_{S}, \hat{\mu}_{A}, \hat{\mu}, \hat{\varepsilon}_{A}, \hat{\varepsilon}_{S}, \hat{\varepsilon}_{r}$ are estimated according to Theorem 4 . Then a simple and reasonably accurate estimate of the quotient $\frac{M_{A r}^{\prime}}{M}$ can be obtained as

$$
\begin{aligned}
\frac{\hat{M}_{A r}^{\prime}}{\hat{M}} & =\frac{1}{6}\left(\frac{1}{\hat{\theta}_{11}}+\hat{\theta}_{12}\right) \frac{\hat{K}_{S}}{\hat{K}_{A}} \frac{\hat{\mu}_{A}}{\hat{\mu}} \\
& +\frac{1}{6}\left(\frac{1}{\hat{\theta}_{31}}+\frac{1}{\hat{\theta}_{33}} \frac{\hat{\varepsilon}_{A}}{\hat{K}_{A}}\right) \frac{\hat{K}_{S}}{\hat{\mu}} \\
& +\frac{1}{6}\left(\hat{\theta}_{32}+\hat{\theta}_{34} \frac{\hat{K}_{S}}{\hat{\varepsilon}_{S}}\right) \frac{\hat{\mu}_{A}}{\hat{K}_{A}}
\end{aligned}
$$

Note that (15) is the mean value of the corresponding solutions for $\frac{\hat{M}_{A r}^{\prime}}{\hat{M}}$ from the static states $\left(\theta_{1}, \theta_{3}\right)$ remaining after the method of Theorem 4 has been used, see (4). Then all components of $\hat{\theta}$ have been utilized for parameter estimation.

After employing Theorem 4, relevant conventional IM parameters can thereafter be estimated by (see [9])

$$
\begin{aligned}
& \hat{L}_{A}^{\prime}=\frac{2}{3} \frac{u_{m}}{\hat{K}_{A}} ; \hat{L}_{S}^{\prime}=\frac{2}{3} \frac{u_{m}}{\hat{K}_{S}} ; \hat{\tau}_{A}=\frac{2}{3} \frac{1}{\hat{\varepsilon}_{A}} ; \\
& \hat{\tau}_{S}=\frac{2}{3} \frac{1}{\hat{\varepsilon}_{S}} ; \hat{\tau}_{r}=\frac{2}{3} \frac{1}{\hat{\varepsilon}_{r}}
\end{aligned}
$$

Additionally, the stator resistances in the three-axis framework are estimated as

$$
\hat{R}_{A}=u_{m}\left(\frac{3}{2} \frac{\hat{\varepsilon}_{A}}{\hat{K}_{A}}-\frac{1}{2} \frac{\hat{\varepsilon}_{S}}{\hat{K}_{S}}\right) ; \hat{R}_{S}=u_{m} \frac{\hat{\varepsilon}_{S}}{\hat{K}_{S}}
$$

\section{OBSERVABILITY/CONTROLlability CONDITIONS}

In the context of observer-based parameter estimation, the sufficient excitation condition of system identification manifests itself in the observer design procedure as an observability condition. Two basic observations can be made.

Firstly, Q should be chosen so that $\{(\mathbf{C}, \mathbf{A})$ is uniformly observable pair $\} \Leftrightarrow\{(\mathbf{H}, \mathbf{A})$ is uniformly observable pair $\}$, to avoid further complications with the Riccati equation.

Secondly, as previously mentioned, $\mathbf{G}$ can be arbitrarily selected. If one uses Part I of Theorem 2, then $(\mathbf{A}, \mathbf{G})$ should be a uniformly controllable pair and the excitation problem reduces to a pure observability problem. The following Lemma gives an aid in choosing $\mathbf{G}$.

Lemma 1: If $\operatorname{rank}\{\mathbf{G}\}=n, t \in \mathcal{I}$, then $(\mathbf{A}, \mathbf{G})$ is an uniformly controllable pair on $\mathcal{I}$.

Proof: Omitted.

If Part II of Theorem 2 is used, then one can choose $\mathbf{G}=\mathbf{0}$.

It is interesting to analyze whether and under what circumstances system (8) becomes unobservable on $\mathcal{I}$.

\section{Lemma 2 (Unobservable Error Subspace):}

Consider system (10) and define $e\left(t_{0}\right)=e_{0}$. Let $\mathcal{E}=\left\{e_{0}: r(t)=0, t \in \mathcal{I}\right\}$. Then the following holds

$$
\{(10) \text { is unobservable on } \mathcal{I}\} \Leftrightarrow \mathcal{E} \neq\{0\}
$$

Proof: See [9].

Lemma 2 proves that analyzing unobservability on $\mathcal{I}$ of a system is equivalent to examining the conditions under which the residual $r$ is identically zero on the considered interval. The following Lemma is also useful in what follows. For simplicity, it utilizes the special structure of the error system (7).

Lemma 3: Assume that (10) has the structure

$$
\begin{aligned}
\dot{e}_{1} & =\mathbf{A}_{\mathbf{1 1}} e_{1}+\mathbf{A}_{\mathbf{1 2}} e_{2}+\mathbf{K}_{\mathbf{1}} r \\
\dot{e}_{2} & =\mathbf{A}_{\mathbf{2 1}} e_{1}+\mathbf{A}_{\mathbf{2 2}} e_{2}+\mathbf{K}_{\mathbf{2}} r \\
r & =e_{1}
\end{aligned}
$$

Assume that $r\left(t_{0}\right)=0$. Then $r(t)=0, t \in \mathcal{I}$ iff

$$
0=\mathbf{A}_{\mathbf{1 2}}(t) \boldsymbol{\Phi}_{\mathbf{2 2}}\left(t, t_{0}\right) e_{2}\left(t_{0}\right), t \in \mathcal{I}
$$

where $\boldsymbol{\Phi}_{\mathbf{2 2}}\left(t, t_{0}\right)$ is the transition matrix for the subsystem $\dot{e}_{2}=\mathbf{A}_{\mathbf{2 2}} e_{2}$. The set $\left\{e_{2}(t)=\mathbf{\Phi}_{\mathbf{2 2}}\left(t, t_{0}\right) e_{2}\left(t_{0}\right): 0=\right.$ 
$\left.\mathbf{A}_{\mathbf{1 2}}(t) e_{2}(t), t \in \mathcal{I}\right\}$ is called the unobservable error subspace on $\mathcal{I}$.

Proof: See [9].

The following Theorem gives explicit expressions corresponding to (19) for system (7). For ease of notation, let $\tilde{z}_{i}^{0}=\tilde{z}_{i}\left(t_{0}\right)$ and $\tilde{\theta}_{i j}^{0}=\tilde{\theta}_{i j}\left(t_{0}\right)$.

Theorem 5 (IM with Faulty Stator): For (7), assume that $r\left(t_{0}\right)=0$. Then $r(t)=0, t \in \mathcal{I}$ iff

$$
\begin{aligned}
0 & =\bar{\omega}(t) \tilde{z}_{4}^{0}+\tilde{z}_{5}^{0} \\
& -\bar{\omega}(t) \imath_{\beta S}(t) \tilde{\theta}_{12}^{0}+u_{\alpha S}(t) \tilde{\theta}_{21}^{0}-\imath_{\alpha S}(t) \tilde{\theta}_{23}^{0} \\
& +\bar{\omega}(t) \int_{t_{0}}^{t} u_{\beta S}(\tau) d \tau \tilde{\theta}_{32}^{0}-\bar{\omega}(t) \int_{t_{0}}^{t} \imath_{\beta S}(\tau) d \tau \tilde{\theta}_{34}^{0} \\
& +\int_{t_{0}}^{t} u_{\alpha S}(\tau) d \tau \tilde{\theta}_{41}^{0}-\int_{t_{0}}^{t} \imath_{\alpha S}(\tau) d \tau \tilde{\theta}_{43}^{0} \\
0 & =-\bar{\omega}(t) \tilde{z}_{3}^{0}+\tilde{z}_{6}^{0} \\
& +\bar{\omega}(t) \imath_{\alpha S}(t) \tilde{\theta}_{11}^{0}+u_{\beta S}(t) \tilde{\theta}_{22}^{0}-\imath_{\beta S}(t) \tilde{\theta}_{24}^{0} \\
& -\bar{\omega}(t) \int_{t_{0}}^{t} u_{\alpha S}(\tau) d \tau \tilde{\theta}_{31}^{0}+\bar{\omega}(t) \int_{t_{0}}^{t} \imath_{\alpha S}(\tau) d \tau \tilde{\theta}_{33}^{0} \\
& +\int_{t_{0}}^{t} u_{\beta S}(\tau) d \tau \tilde{\theta}_{42}^{0}-\int_{t_{0}}^{t} \imath_{\beta S}(\tau) d \tau \tilde{\theta}_{44}^{0}
\end{aligned}
$$

$t \in \mathcal{I}$.

Proof: Follows from Lemma 3.

\section{Simulation Results}

To support the preceding theoretical results, simulations were performed. In fact, this is the only way to validate a parameter estimation algorithm since in real world the actual values of IMs are not known and also depend on experimental conditions.

The model parameters for the electrical part were chosen as those identified for actual IMs in [9]. It was assumed that all relevant variables were perfectly known, $i$. $e$. the electrical rotor speed $\bar{\omega}$, the two stator currents $\left(\imath_{\alpha S}\right.$ and $\left.\imath_{\beta S}\right)$ and the two normalized stator voltages $\left(\bar{u}_{\alpha S}\right.$ and $\left.\bar{u}_{\beta S}\right)$. The normalized stator voltages were assumed to be obtained from a perfectly balanced voltage source leading to

$$
\begin{aligned}
& \bar{u}_{\alpha S}=\cos \left(\omega_{s}[t-0.5]\right) \\
& \bar{u}_{\beta S}=\sin \left(\omega_{s}[t-0.5]\right)
\end{aligned}
$$

where $\omega_{s}=2 \pi f=100 \pi$, i.e. a voltage source with net frequency $f=50$. These voltages were selected in order to resemble a symmetrical 3-phase mains with amplitude $\bar{u}_{m}=$ $75 \mathrm{~V}$ and normalization factor $u_{m}=75 \mathrm{~V}$. These values correspond to the ones used in experiments mentioned earlier.

For all simulations, $\mathbf{Q}=\gamma^{2} \mathbf{I}, \mathbf{G}=\gamma \mathbf{C}^{H}, \mathbf{P}(0)=10^{5} \mathbf{I}$ and $\alpha=0.2$ where $\gamma$ was chosen in a suitable manner for each situation. For an illustration of the effects of different choices of the design degrees of freedom, see [10].

The mechanical part is simulated as well,

$$
\dot{\bar{\omega}}=-\alpha_{m} \bar{\omega}+\delta\left(\bar{M}_{e m}-\bar{M}_{l}\right)
$$

where the expressions for the normalized electromechanical torque $\bar{M}_{e m}$ and the normalized load $\bar{M}_{l}$ depend on the situation in hand. All further details can be found in [9].
In the sequel, $\alpha_{m}=0.1, \delta=4 \cdot 10^{6}$, chosen by trial and error to resemble the behavior of a real-life IM.

All simulations were performed in such a way that they can be verified in a similar manner in a simple experimental setup later on. The voltage source was switched on at $t=0.5$ $\mathrm{s}$, in order to simulate a start-up. In cases were it applies, the mechanical load was switched on at $t=0.5 \mathrm{~s}$ as well.

Two different kinds of simulations were performed in order to test different excitation conditions.

- Sinusoidal Load - The mechanical load was varied according to $\bar{M}_{l}=7.5 \cdot 10^{-6}\left(1-\cos \left(\frac{1}{3} \pi[t-0.5]\right)\right)$, simulating a load varying with a period of $6 \mathrm{~s}$.

- Switched Voltage Source - The voltage source was switched on and off in alternating intervals of $3 \mathrm{~s}$ respectively, whereas $\bar{M}_{l}=0$.

Here model $(1,2)$ and the observer $(6)$ where $\mathbf{K}$ is defined by (9) were used. The system matrices are given by (8). For this case, the normalized electromechanical torque is described by the algebraic relationships

$$
\begin{aligned}
& \bar{M}_{e m}=k_{1}\left[\bar{\psi}_{\beta S} \bar{x}_{\alpha S}-\bar{\psi}_{\alpha S} \bar{x}_{\beta S}-\left(1-\frac{\mu}{\mu_{A}}\right) \bar{x}_{\alpha S} \bar{x}_{\beta S}\right] \\
& k_{1}=\frac{M}{M_{A r}^{\prime}} \frac{1-\mu_{A}}{\mu_{A}} ; \bar{\imath}_{\alpha S}=\frac{1}{\mu_{A}} \bar{\psi}_{\alpha S}-\frac{\mu}{\mu_{A}} \frac{1-\mu_{A}}{\mu_{A}} \bar{x}_{\alpha S} \\
& \bar{\imath}_{\beta S}=\frac{1}{\mu} \bar{\psi}_{\beta S}-\frac{1-\mu}{\mu} \bar{x}_{\beta S}
\end{aligned}
$$

The design parameter was $\gamma=10^{4}$ during simulations.

\section{A. Inter-turn Short Circuit}

Here the parameters were the same as the parameters identified in [9] when there was an inter-turn short circuit in one stator phase, see Table I. The three-axis stator resistances

\begin{tabular}{|l|l||l|l|}
\hline \hline \multicolumn{2}{|c||}{ Fault invariant parameter } & \multicolumn{2}{|c|}{ Fault influenced parameter } \\
\hline Parameter & Value & Parameter & Value \\
\hline$L_{S}^{\prime}$ & 0.4434 & $L_{A}^{\prime}$ & 0.1407 \\
$\mu$ & 0.08057 & $\mu_{A}$ & 0.2175 \\
$\tau_{S}$ & 0.05430 & $\tau_{A}$ & 0.01487 \\
$\tau_{r}$ & 0.07761 & $\frac{M_{A r}^{\prime}}{M}$ & 0.8471 \\
\hline \hline
\end{tabular}

TABLE I

PARAMETER VALUES FOR IM WITH INTER-TURN SHORT CIRCUIT

can be calculated as $R_{A}=10.12$ and $R_{S}=8.165$. In order to simulate a sudden fault in the IM, the initial values are chosen according to those values obtained for the healthy IM. The initial estimated stator resistances are estimated as $\hat{R}_{A}\left(t_{0}\right)=10.15$ and $\hat{R}_{S}\left(t_{0}\right)=9.393$.

The estimates of $R_{A}$ and $R_{S}$ for sinusoidal load are given in Fig. 1 and for switching power source in Fig. 2

\section{DISCUSSION}

Considering the simulation results, it can be concluded that no complex experimental arrangements have to be used in order to assure sufficient excitation of the system. A single-tone voltage source is sufficient. Remarkably, even such weak perturbation as a varying load assures that excitation is rich enough. Due to the fact that no superfluous 


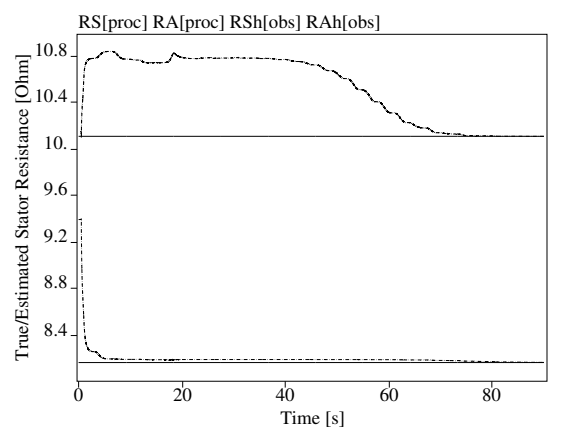

Fig. 1. Identification of an IM with inter-turn short circuit. Excitation by sinusoidal load. $R_{A}$ and $R_{S}$ are depicted. Solid lines - Actual parameter values. Dotted lines - Estimated parameters. Stator voltage is switched on at $0.5 \mathrm{~s}$.

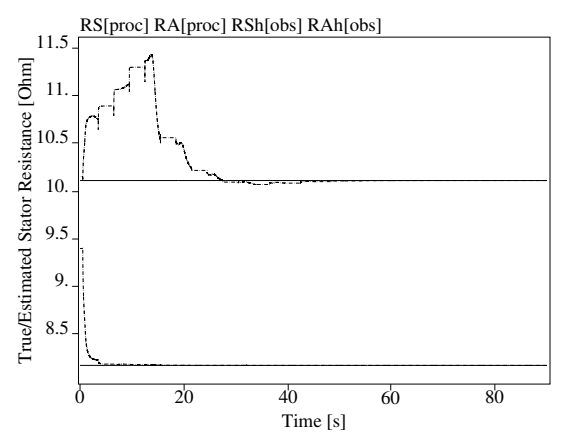

Fig. 2. Identification of an IM with inter-turn short circuit. Excitation by switched voltage source. $R_{A}$ and $R_{S}$ are depicted. Solid lines - Actual parameter values. Dotted lines - Estimated parameters. Stator voltage is switched on at $0.5 \mathrm{~s}$.

assumptions have been made, all electrical parameters were successfully identified in each case considered. For example, one commonly accepted assumption is that the rotor speed is approximately constant, which obviously does not hold and is not needed in this case.

There are several possible ways of proceeding from this point. Obviously, an absolute necessity is to validate the algorithm on a real IM in order to confirm the promising simulation results.

Another one is to reduce the complexity of the observer as much as possible, $i$. e. expanding all matrix differential equations into scalar ones. Due to the sparsity of the matrices involved, significant simplification can be expected. A main step in this direction is to use simple structures for $\mathbf{Q}$ and G.

A very interesting topic is relaxing the need for linear system description. A non-linear design, although more complex for analysis, offers several benefits. One of them is a lower differential order of the resulting observer, since the redundant states are removed. Less problems with poor excitation are also expected, due to the nonlinearities.

Another important topic for further research is to investigate how measurement noise affects the identification results. It is likely that measurement noise can seriously deteriorate the results, due to unfavorable redundancy in the observer.

To proceed, the theoretical results should be extended to IMs with rotor fault as well. However, this is not straight- forward because unknown trigonometric transformations depending on the rotor angle appear in the model.

Nevertheless, despite all questions remaining to be answered, a foundation for simultaneous on-line identification of all parameters in IMs has been laid. At present, the presented method can be used for healthy IMs and for IMs with stator faults. The suggested approach has potential both in accurate parameter estimation as well as fault detection of IMs under stator fault, at the same time indicating which fault is present and to what extent. The main strength of the methodology is that no differentiation of measured signals is needed, and the common assumption of approximately constant rotor speed can be successfully avoided.

\section{CONCLUSION}

In this paper, a recently derived model for IMs subject to stator faults is used for identification of all electrical parameters simultaneously and on-line. The model is augmented with static parameter states and analytical conditions regarding sufficient for parameter estimation excitation are provided. Specifically, conditions for the practically important case of IMs running at stationary conditions were also derived. Simulations show that single-tone stator voltages are sufficient for successful identification of all electrical parameters. Using such an input and a time-varying load was proven to provide sufficiently rich excitation.

\section{REFERENCES}

[1] P. J. R. de Oliveira and Z. M. A. Peixoto, "Parameter estimation of a induction machine using a continuous time model," in 24th Annual Conference of the IEEE Industrial Electronics Society IECON, 1998.

[2] M. Cirrincione and M. Pucci, "A direct torque-control of an ac drive based on a recursive-least-square (RLS) method," in International Symposium on Diagnostics for Electrical Machines, Power Electronics and Drives SDEMPED, 2001.

[3] M. Cirrincione, M. Pucci, G. Cirrincione, and G.-A. Capolino, "A new experimental application of least-squares techniques for the estimation of the induction motor parameters," IEEE Transactions on Industry Applications, vol. 39, no. 5, September/October 2003.

[4] L. A. S. Ribeiro, C. B. Jacobina, and A. M. N. Lima, "The influence of the slip and the speed in the parameter estimation of induction machines," in 28th Annual IEEE Power Electronics Specialists Conference PESC, 1997.

[5] V. Elfdem, A. J. Wetherilt, S. Parmaksiz, E. Albas, H. Cecen, and A. Duyar, "On the identification of three phase induction motors," in European Control Conference ECC, 1999.

[6] G. Besancon, "On-line full state and parameter estimation in induction motors and application in control and monitoring," in European Control Conference ECC, 2001.

[7] M. Stocks, F. Rodyukov, and A. Medvedev, "Idealized two-axis model of induction machines under stator fault," in 1st IEEE Conference on Industrial Electronics and Applications ICIEA, Singapore, 2006.

[8] M. Stocks, "Modeling, analysis, state and parameter estimation of faulty induction machines," $\mathrm{Ph}$. D. Thesis, Luleå University of Technology, Sweden, October 2006.

[9] M. Stocks and A. Medvedev, "On-line estimation of all electrical parameters in symmetrical and faulty induction machines: Stator fault case," CSEE / Control Engineering Group, Tech. Rep. ISSN 14021536 / ISRN LTU-TR-06/10-SE / NR 2006:10, 2006.

[10] — , "Guaranteed convergence rate for linear-quadratic optimal timevarying observers," in IEEE Conference on Decision and Control, San Diego, CA, 2006. 